\title{
Transverse impedance of a periodic array of cavities
}

\author{
A. V. Fedotov, R. L. Gluckstern, and M. Venturini \\ Physics Department, University of Maryland, College Park, Maryland 20742
}

(Received 19 March 1999; published 21 June 1999)

\begin{abstract}
We examine the transverse impedance of a periodic array of cavities in a beam pipe at high frequency. The calculation is an extension of a previous one for the longitudinal impedance of a periodic array of azimuthally symmetric pillboxes, for which only TM modes were needed. In the present case, we must include TE modes as well. In addition, we extend the applicability of the previous calculation by including an extra term in the coupling kernel so that the results are valid for all values of the ratio of the cavity length to the period of the structure (all values of the ratio of iris thickness to structure period). In spite of the presence of TE modes, we find that the high frequency limit of the transverse impedance is simply $\left(2 / k a^{2}\right)$ times the corresponding limit of the longitudinal impedance, just as it is for the resistive wall impedances, a relation which occurs frequently for azimuthally symmetric structures. Finally, we present numerical results as well as approximate expressions for the impedance per period, valid for all ratios of cavity length to structure period. [S1098-4402(99)00045-2]
\end{abstract}

PACS numbers: 29.27.Bd, 41.20.-q

\section{INTRODUCTION}

The acceleration of charged particles in periodic structures leads to wakefields which are capable of interacting adversely with particles in the same bunch, or in following bunches. The conventional method of describing these limitations in the current which can be accelerated is the longitudinal and transverse coupling impedances of the structure. With the increasing use of short bunches, it becomes necessary to evaluate these coupling impedances at high frequencies (wavelength of the order of the bunch length). This has been done for the longitudinal impedance of small periodic obstacles in an azimuthally symmetric structure [1,2], where we have derived an integral equation for the axial electric field at the inner bore radius. By taking the high frequency limit of the kernel in the integral equation, it was found that the local frequency average of the real part of the longitudinal impedance per period of the structure varies as the $-3 / 2$ power of the frequency.

In this paper we address the corresponding problem for the transverse coupling impedance at high frequency. In particular, we first derive the integral equation for the electric field at the bore radius of the structure and obtain the result for the transverse impedance of a single small obstacle at high frequency by way of the high frequency limit of the kernels. The problem is more complicated than it was for the longitudinal impedance, since both TM and TE waveguide modes are present, which requires matching two components of the magnetic field at the boundary between the waveguide and the obstacle. However, as we shall eventually see, in the high frequency limit the TM contribution dominates for a single obstacle, but the TE contributions must be included in the periodic case. Nevertheless, we find the same relation between the longitudinal and transverse impedances as exists for the resistive wall impedances of a circular beam pipe.

\section{ANALYSIS OF PIPE FIELDS FOR A SINGLE PILLBOX OBSTACLE [3]}

We consider a point charge $Q$ traveling in the $z$ direction at $(x, y)=\left(\Delta_{x}, 0\right)$. We start with the definition of the $x$ component of the transverse coupling impedance as a function of $k=\omega / c$. In the limit of small $\Delta_{x}$, we have

$$
Z_{x}(k)=\frac{j}{Q \Delta_{x}} \int_{-\infty}^{\infty} d z e^{j k z}\left[E_{x}(z ; k)-Z_{0} H_{y}(z ; k)\right]_{\substack{x=0 \\ y=0}}
$$

in units of $[\Omega / \mathrm{m}]$ (of transverse displacement). Here $Z_{0}=$ $\sqrt{\mu_{0} / \epsilon_{0}}=120 \pi[\Omega]$ is the impedance of free space. We then use

$$
\frac{\partial E_{x}}{\partial z}-\frac{\partial E_{z}}{\partial x}=-j k Z_{0} H_{y}
$$

to obtain the alternate expression

$$
Z_{x}(k)=-\left.\frac{1}{Q \Delta_{x} k} \int_{-\infty}^{\infty} d z e^{j k z} \frac{\partial E_{z}}{\partial x}\right|_{x=0, y=0},
$$

where we use the time dependence $\exp (j \omega t)$. The geometry is shown in Fig. 1. At ultrarelativistic velocity, the source field components in the frequency domain, for $r \gg \Delta_{x}$, are

$$
\begin{aligned}
E_{r}^{(s)}(r, \theta, z ; k) & =Z_{0} H_{\theta}^{(s)}(r, \theta, z ; k) \\
& =\frac{Q \Delta_{x} Z_{0}}{2 \pi}\left(\frac{1}{r^{2}}+\frac{1}{a^{2}}\right) e^{-j k z} \cos \theta, \\
E_{\theta}^{(s)}(r, \theta, z ; k) & =-Z_{0} H_{r}^{(s)}(r, \theta, z ; k) \\
& =\frac{Q \Delta_{x} Z_{0}}{2 \pi}\left(\frac{1}{r^{2}}-\frac{1}{a^{2}}\right) e^{-j k z} \sin \theta, \\
E_{z}^{(s)}(r, \theta, z ; k) & =0, \quad H_{z}^{(s)}(r, \theta, z ; k)=0 .
\end{aligned}
$$


We now recognize that the field components $E_{z}, E_{r}, H_{\theta}$ will be proportional to $\cos \theta$, and $H_{z}, H_{r}, E_{\theta}$ will be proportional to $\sin \theta$. Suppressing this $\theta$ dependence, we write the field components in terms of TM and TE modes as

$$
\begin{gathered}
E_{z}(r, z ; k)=\int_{-\infty}^{\infty} d q A(q) \frac{J_{1}(\kappa r)}{J_{1}(\kappa a)} e^{-j q z}, \\
E_{r}(r, z ; k)=-j \int_{-\infty}^{\infty} d q\left[A(q) \frac{J_{1}^{\prime}(\kappa r)}{J_{1}(\kappa a)} \frac{q}{\kappa}+B(q) \frac{J_{1}(\kappa r)}{J_{1}^{\prime}(\kappa a)} \frac{k}{r \kappa^{2}}\right] e^{-j q z}+\frac{Q \Delta_{x} Z_{0}}{2 \pi}\left(\frac{1}{r^{2}}+\frac{1}{a^{2}}\right) e^{-j k z}, \\
E_{\theta}(r, z ; k)=j \int_{-\infty}^{\infty} d q\left[A(q) \frac{J_{1}(\kappa r)}{J_{1}(\kappa a)} \frac{q}{r \kappa^{2}}+B(q) \frac{J_{1}^{\prime}(\kappa r)}{J_{1}^{\prime}(\kappa a)} \frac{k}{\kappa}\right] e^{-j q z}+\frac{Q \Delta_{x} Z_{0}}{2 \pi}\left(\frac{1}{r^{2}}-\frac{1}{a^{2}}\right) e^{-j k z}, \\
Z_{0} H_{r}(r, z ; k)=-j \int_{-\infty}^{\infty} d q\left[A(q) \frac{J_{1}(\kappa r)}{J_{1}(\kappa a)} \frac{k}{r \kappa^{2}}+B(q) \frac{J_{1}^{\prime}(\kappa r)}{J_{1}^{\prime}(\kappa a)} \frac{q}{\kappa}\right] e^{-j q z}-\frac{Q \Delta_{x} Z_{0}}{2 \pi}\left(\frac{1}{r^{2}}-\frac{1}{a^{2}}\right) e^{-j k z}, \\
Z_{0} H_{\theta}(r, z ; k)=-j \int_{-\infty}^{\infty} d q\left[A(q) \frac{J_{1}^{\prime}(\kappa r)}{J_{1}(\kappa a)} \frac{k}{\kappa}+B(q) \frac{J_{1}(\kappa r)}{J_{1}^{\prime}(\kappa a)} \frac{q}{r \kappa^{2}}\right] e^{-j q z}+\frac{Q \Delta_{x} Z_{0}}{2 \pi}\left(\frac{1}{r^{2}}+\frac{1}{a^{2}}\right) e^{-j k z} .
\end{gathered}
$$

Here $\kappa^{2} \equiv k^{2}-q^{2}$ and the contour in the complex $q$ plane goes below the poles at $J_{1}(\kappa a)=0$ (TM propagating modes) and $J_{1}^{\prime}(\kappa a)=0$ (TE propagating modes) on the negative real $q$ axis and above the corresponding poles on the positive real $q$ axis. This ensures that the stimulated waveguide modes are outgoing from the obstacle.

We now note that $E_{z}(z) \equiv E_{z}(a, z ; k)$ and $E_{\theta}(z) \equiv$ $E_{\theta}(a, z ; k)$ vanish except for $0 \leq z \leq g$ for a perfectly conducting pipe wall. This enables us to obtain expressions for $A(q)$ and $B(q)$ in terms of these field components by taking the inverse Fourier transforms of Eqs. (2.7) and (2.9). Specifically, we find

$$
A(q)=\frac{1}{2 \pi} \int_{0}^{g} d z E_{z}(z) e^{j q z}
$$

and

$$
\frac{q A(q)}{\kappa^{2} a}+\frac{k}{\kappa} B(q)=-\frac{j}{2 \pi} \int_{0}^{g} d z E_{\theta}(z) e^{j q z} .
$$

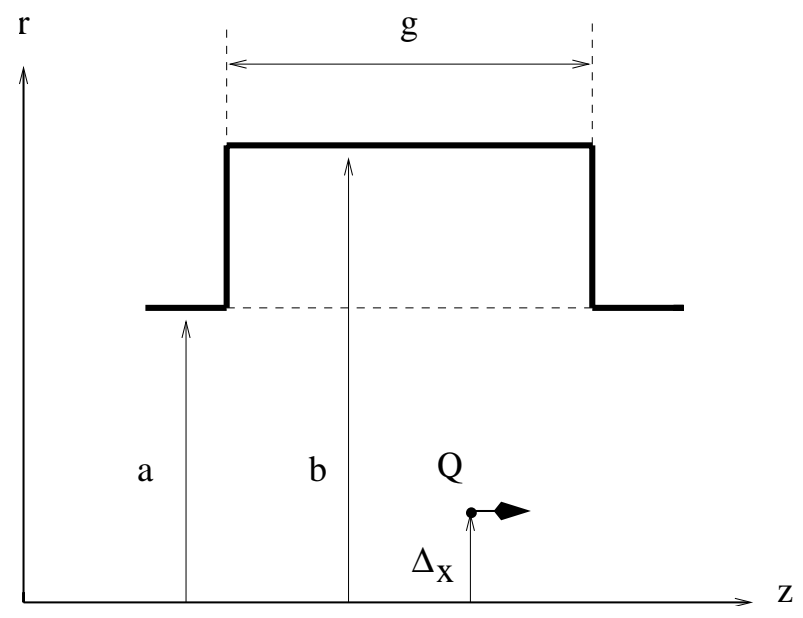

FIG. 1. The layout of geometry for a single cavity.
We now express $H_{\theta}(z)$ and $H_{z}(z)$ at the pipe radius, which will later be matched to the corresponding fields in the cavity, in terms of $E_{z}(z)$ and $E_{\theta}(z)$ at the pipe radius. Specifically, we define

$$
\begin{gathered}
Z_{0} H_{\theta}(z) \equiv \frac{Q \Delta_{x} Z_{0}}{\pi a^{2}} e^{-j k z} h_{\theta}(z), \\
Z_{0} H_{z}(z) \equiv \frac{Q \Delta_{x} Z_{0}}{\pi a^{2}} e^{-j k z} h_{z}(z), \\
E_{z}(z) \equiv \frac{Q \Delta_{x} Z_{0}}{\pi a^{2}} e^{-j k z} e_{z}(z), \\
E_{\theta}(z) \equiv \frac{Q \Delta_{x} Z_{0}}{\pi a^{2}} e^{-j k z} e_{\theta}(z),
\end{gathered}
$$

and obtain

$$
\begin{gathered}
h_{\theta}(z)=-\int_{0}^{g} d z^{\prime}\left[\mathcal{P}_{11}^{p}\left(z-z^{\prime}\right)+\mathcal{Q}_{11}^{p}\left(z-z^{\prime}\right)\right] e_{z}\left(z^{\prime}\right) \\
-\int_{0}^{g} d z^{\prime} \mathcal{Q}_{12}^{p}\left(z-z^{\prime}\right) e_{\theta}\left(z^{\prime}\right)+1 \\
h_{z}(z)=-\int_{0}^{g} d z^{\prime} \mathcal{Q}_{21}^{p}\left(z-z^{\prime}\right) e_{z}(z) \\
-\int_{0}^{g} d z^{\prime} \mathcal{Q}_{22}^{p}\left(z-z^{\prime}\right) e_{\theta}\left(z^{\prime}\right)
\end{gathered}
$$

Here we define $u=z-z^{\prime}$ and obtain

$$
\begin{aligned}
& \mathcal{P}_{11}^{p}(u)=\frac{j}{2 \pi} \int_{-\infty}^{\infty} d q \frac{k}{\kappa} \frac{J_{1}^{\prime}(\kappa a)}{J_{1}(\kappa a)} e^{-j q u+j k u}, \\
& \mathcal{Q}_{11}^{p}(u)=-\frac{j}{2 \pi} \int_{-\infty}^{\infty} d q \frac{q^{2}}{k \kappa^{3} a^{2}} \frac{J_{1}(\kappa a)}{J_{1}^{\prime}(\kappa a)} e^{-j q u+j k u},
\end{aligned}
$$




$$
\begin{gathered}
\mathcal{Q}_{12}^{p}(u)=\frac{1}{2 \pi} \int_{-\infty}^{\infty} d q \frac{q}{k \kappa a} \frac{J_{1}(\kappa a)}{J_{1}^{\prime}(\kappa a)} e^{-j q u+j k u}=\mathcal{Q}_{21}^{p}(u), \\
\mathcal{Q}_{22}^{p}(u)=\frac{j}{2 \pi} \int d q \frac{\kappa}{k} \frac{J_{1}(\kappa a)}{J_{1}^{\prime}(\kappa a)} e^{-j q u+j k u} .
\end{gathered}
$$

Finally, for reasons of convergence, we integrate the last term in Eq. (2.20) by parts with respect to $z^{\prime}$, setting $e_{\theta}(0)=e_{\theta}(g)=0$, and obtain

$$
\begin{aligned}
h_{z}(z)= & -\int_{0}^{g} d z^{\prime} \mathcal{Q}_{21}^{p}\left(z-z^{\prime}\right) e_{z}\left(z^{\prime}\right) \\
& -\int_{0}^{g} d z^{\prime} \frac{d e_{\theta}\left(z^{\prime}\right)}{d z^{\prime}} \tilde{\mathcal{Q}}_{22}^{p}\left(z-z^{\prime}\right),
\end{aligned}
$$

where

$$
\tilde{Q}_{22}^{p}(u)=\frac{1}{2 \pi} \int_{-\infty}^{\infty} d q \frac{(k+q)}{k \kappa} \frac{J_{1}(\kappa a)}{J_{1}^{\prime}(\kappa a)} e^{-j q u+j k u} .
$$

The kernels denoted by $\mathcal{P}(u)$ come from TM modes and those denoted by $\mathcal{Q}(u)$ come from TE modes. Their evaluation is continued in Appendix A, leading to the results in Eqs. (A10), (A13), (A15), and (A16).

\section{ANALYSIS OF FIELDS IN PILLBOX CAVITY}

We expand the fields in the cavity region $(0 \leq z \leq g$, $a \leq r \leq b$ ) for frequency $k c / 2 \pi$ as a Fourier series in $z$. In particular, we write, for the TM modes,

$$
E_{z}(r, z)=\sum_{n=0}^{\infty} a_{n} \cos \left(\frac{n \pi z}{g}\right) \frac{F(\sigma r)}{F(\sigma a)},
$$

with

$$
\sigma^{2}=k^{2}-(n \pi / g)^{2}
$$

and

$$
F(u)=H_{+}(u)-\lambda H_{-}(u),
$$

where $H_{ \pm}(u) \equiv H_{1}^{(1,2)}(u)$ are the Hankel functions. The parameter $\lambda$ is chosen so that $F(\sigma b)=0$; that is

$$
\lambda=\frac{H_{+}(\sigma b)}{H_{-}(\sigma b)} .
$$

The form in Eq. (3.1) clearly satisfies $\left(\nabla^{2}+k^{2}\right) E_{z}=0$ and $E_{z}(b, z)=0$, as required by the boundary conditions [4].

For the TE modes we similarly write

$$
Z_{0} H_{z}(r, z)=\sum_{n=0}^{\infty} b_{n} \sin \left(\frac{n \pi z}{g}\right) \frac{G(\sigma r)}{G^{\prime}(\sigma a)},
$$

with

$$
G(u)=H_{+}(u)-\mu H_{-}(u) .
$$

The parameter $\mu$ is chosen so that $G^{\prime}(\sigma b)=0$; that is [4]

$$
\mu=\frac{H_{+}^{\prime}(\sigma b)}{H_{-}^{\prime}(\sigma b)} .
$$

The remaining field components can then be written as

$$
\begin{gathered}
E_{r}(r, z)=\sum_{n=0}^{\infty} \sin \left(\frac{n \pi z}{g}\right)\left[-\frac{n \pi a_{n}}{g \sigma} \frac{F^{\prime}(\sigma r)}{F(\sigma a)}-\frac{j k b_{n}}{\sigma^{2} r} \frac{G(\sigma r)}{G^{\prime}(\sigma a)}\right], \\
E_{\theta}(r, z)=\sum_{n=0}^{\infty} \sin \left(\frac{n \pi z}{g}\right)\left[\frac{n \pi a_{n}}{g \sigma^{2} r} \frac{F(\sigma r)}{F(\sigma a)}+\frac{j k b_{n}}{\sigma} \frac{G^{\prime}(\sigma r)}{G^{\prime}(\sigma a)}\right], \\
Z_{0} H_{r}(r, z)=\sum_{n=0}^{\infty} \cos \left(\frac{n \pi z}{g}\right)\left[-\frac{j k a_{n}}{\sigma} \frac{F(\sigma r)}{F(\sigma a)}+\frac{n \pi b_{n}}{g \sigma^{2} r} \frac{G(\sigma r)}{G^{\prime}(\sigma a)}\right], \\
Z_{0} H_{\theta}(r, z)=\sum_{n=0}^{\infty} \cos \left(\frac{n \pi z}{g}\right)\left[-\frac{j k a_{n}}{\sigma} \frac{F^{\prime}(\sigma r)}{F(\sigma a)}+\frac{n \pi b_{n}}{g \sigma^{2} r} \frac{G(\sigma r)}{G^{\prime}(\sigma a)}\right] .
\end{gathered}
$$

The tangential electric field components at $r=a$ are

$$
\begin{gathered}
E_{z}(a, z) \equiv E_{z}(z)=\sum_{n=0}^{\infty} a_{n} \cos \left(\frac{n \pi z}{g}\right), \\
E_{\theta}(a, z) \equiv E_{\theta}(z)=\sum_{n=0}^{\infty} \sin \left(\frac{n \pi z}{g}\right)\left[\frac{n \pi a_{n}}{g \sigma^{2} a}+\frac{j k b_{n}}{\sigma}\right],
\end{gathered}
$$

from which one can obtain the coefficients $a_{n}$ and $b_{n}$ as

$$
a_{n}=\frac{2}{g\left(1+\delta_{0 n}\right)} \int_{0}^{g} d z E_{z}(z) \cos \left(\frac{n \pi z}{g}\right)
$$




$$
b_{n}=\frac{2 \sigma}{j k g} \int_{0}^{g} d z E_{\theta}(z) \sin \left(\frac{n \pi z}{g}\right)-\frac{2 n \pi}{j k g^{2} a \sigma\left(1+\delta_{0 n}\right)} \int_{0}^{g} d z E_{z}(z) \cos \left(\frac{n \pi z}{g}\right) .
$$

We now express the tangential magnetic fields at $r=a$ in terms of the tangential electric fields at $r=a$ by combining Eqs. (3.10), (3.11), (3.14), and (3.15). Using the definitions in Eqs. (2.15)-(2.18), we obtain

$$
\begin{gathered}
h_{\theta}(z)=\int_{0}^{g} d z^{\prime}\left[\mathcal{P}_{11}^{c}\left(z, z^{\prime}\right)+\mathcal{Q}_{11}^{c}\left(z, z^{\prime}\right)\right] e_{z}\left(z^{\prime}\right)+\int_{0}^{g} d z^{\prime} \mathcal{Q}_{12}^{c}\left(z, z^{\prime}\right) e_{\theta}\left(z^{\prime}\right), \\
h_{z}(z)=\int_{0}^{g} d z^{\prime} \mathcal{Q}_{21}^{c}\left(z, z^{\prime}\right) e_{z}\left(z^{\prime}\right)+\int_{0}^{g} d z^{\prime} \mathcal{Q}_{22}^{c}\left(z, z^{\prime}\right) e_{\theta}\left(z^{\prime}\right),
\end{gathered}
$$

where

$$
\begin{gathered}
\mathcal{P}_{11}^{c}\left(z, z^{\prime}\right)=\sum_{n=0}^{\infty} \frac{-2 j k a e^{j k\left(z-z^{\prime}\right)}}{g\left(1+\delta_{0 n}\right)} \cos \left(\frac{n \pi z}{g}\right) \cos \left(\frac{n \pi z^{\prime}}{g}\right) \frac{F^{\prime}(\sigma a)}{\sigma a F(\sigma a)} \\
\mathcal{Q}_{11}^{c}\left(z, z^{\prime}\right)=\sum_{n=0}^{\infty} \frac{2 j}{k g a}\left(\frac{n \pi}{g \sigma}\right)^{2} \frac{e^{j k\left(z-z^{\prime}\right)}}{\left(1+\delta_{0 n}\right)} \cos \left(\frac{n \pi z}{g}\right) \cos \left(\frac{n \pi z^{\prime}}{g}\right) \frac{G(\sigma a)}{\sigma a G^{\prime}(\sigma a)}, \\
\mathcal{Q}_{12}^{c}\left(z, z^{\prime}\right)=-\sum_{n=0}^{\infty} \frac{2 j n \pi}{k g^{2}} e^{j k\left(z-z^{\prime}\right)} \cos \left(\frac{n \pi z}{g}\right) \sin \left(\frac{n \pi z^{\prime}}{g}\right) \frac{G(\sigma a)}{\sigma a G^{\prime}(\sigma a)} \\
\mathcal{Q}_{21}^{c}\left(z, z^{\prime}\right)=\sum_{n=0}^{\infty} \frac{2 j n \pi}{k g^{2}} e^{j k\left(z-z^{\prime}\right)} \sin \left(\frac{n \pi z}{g}\right) \cos \left(\frac{n \pi z^{\prime}}{g}\right) \frac{G(\sigma a)}{\sigma a G^{\prime}(\sigma a)} \\
\mathcal{Q}_{22}^{c}\left(z, z^{\prime}\right)=-\sum_{n=0}^{\infty} \frac{2 j \sigma^{2} a}{k g} e^{j k\left(z-z^{\prime}\right)} \sin \left(\frac{n \pi z}{g}\right) \sin \left(\frac{n \pi z^{\prime}}{g}\right) \frac{G(\sigma a)}{\sigma a G^{\prime}(\sigma a)}
\end{gathered}
$$

Once again, we define $\tilde{Q}_{22}^{c}\left(z, z^{\prime}\right)=-\int d z^{\prime} Q_{22}^{c}\left(z, z^{\prime}\right)$, but shall postpone the evaluation of $\tilde{Q}_{22}^{c}\left(z, z^{\prime}\right)$ until after we obtain the high frequency limit.

\section{INTEGRAL EQUATIONS AND SOLUTION FOR IMPEDANCE}

We now combine Eqs. (2.19) and (2.20) for the pipe region with Eqs. (3.16) and (3.17) for the cavity region by equating the tangential magnetic fields at $r=a$ for $0 \leq z \leq g$. This leads to the integral equations

$$
\begin{gathered}
\int_{0}^{g} d z^{\prime}\left[\mathcal{P}_{11}\left(z, z^{\prime}\right)+\mathcal{Q}_{11}\left(z, z^{\prime}\right)\right] e_{z}\left(z^{\prime}\right)+ \\
\int_{0}^{g} d z^{\prime} \mathcal{Q}_{12}\left(z, z^{\prime}\right) e_{\theta}\left(z^{\prime}\right)=1, \\
\int_{0}^{g} d z^{\prime} \mathcal{Q}_{21}\left(z, z^{\prime}\right) e_{z}\left(z^{\prime}\right)+ \\
\int_{0}^{g} d z^{\prime} \tilde{\mathcal{Q}}_{22}\left(z, z^{\prime}\right) \frac{d e_{\theta}\left(z^{\prime}\right)}{d z^{\prime}}=0,
\end{gathered}
$$

where

$$
\begin{aligned}
& \mathcal{P}\left(z, z^{\prime}\right)=\mathcal{P}^{p}\left(z-z^{\prime}\right)+\mathcal{P}^{c}\left(z, z^{\prime}\right), \\
& \mathcal{Q}\left(z, z^{\prime}\right)=\mathcal{Q}^{p}\left(z-z^{\prime}\right)+\mathcal{Q}^{c}\left(z, z^{\prime}\right) .
\end{aligned}
$$

Our next task is to proceed to the limit of high frequency (large $k$ ) limit, obtaining the smoothed fields (averaged over high frequency oscillations). To do this we first obtain the smoothed high frequency limits of the pipe and cavity kernels. The details are contained in Appendix B for the pipe kernels and in Appendix $\mathrm{C}$ for the cavity kernels. The results are summarized below. All kernels vanish for $u<0$. For $u>0$ we have, for the leading terms for large $k$,

$$
\begin{aligned}
& \mathcal{P}_{11}^{p}(u)+\mathcal{Q}_{11}^{p}(u)=\mathcal{P}_{11}^{c}(u)+\mathcal{Q}_{11}^{c}(u) \\
& \cong-\frac{1+j}{2} \sqrt{\frac{k}{\pi u}}, \\
& \mathcal{Q}_{12}^{p}(u)=\mathcal{Q}_{21}^{p}(u)=\mathcal{Q}_{12}^{c}(u)=\mathcal{Q}_{21}^{c}(u) \\
& \cong \frac{1-j}{2 a} \frac{1}{\sqrt{\pi k u}}, \\
& \tilde{Q}_{22}^{p}(u)=\tilde{Q}_{22}^{c}(u) \cong \frac{1-j}{\sqrt{\pi k u}},
\end{aligned}
$$

with $u=z-z^{\prime}$. Remarkably, the corresponding pipe and cavity kernels are identical for large $k$ after smoothing. The same situation prevailed in our earlier calculation for the smoothed longitudinal coupling impedance at high frequency for a single obstacle with only TM modes being considered [1]. 
Thus the integral equations become [5]

$$
\begin{gathered}
-(1+j) \sqrt{\frac{k}{\pi}} \int_{0}^{z} \frac{d z^{\prime} e_{z}\left(z^{\prime}\right)}{\sqrt{z-z^{\prime}}}+ \\
\frac{1-j}{a \sqrt{\pi k}} \int_{0}^{z} \frac{d z^{\prime} e_{\theta}\left(z^{\prime}\right)}{\sqrt{z-z^{\prime}}}=1, \\
\frac{1-j}{a \sqrt{k \pi}} \int_{0}^{z} \frac{d z^{\prime} e_{z}\left(z^{\prime}\right)}{\sqrt{z-z^{\prime}}}+ \\
\frac{2(1-j)}{\sqrt{\pi k}} \int_{0}^{z} \frac{d z^{\prime}}{\sqrt{z-z^{\prime}}} \frac{d e_{\theta}}{d z^{\prime}}=0 .
\end{gathered}
$$

From Eq. (4.9) we see that, for large $k$,

$$
e_{z}\left(z^{\prime}\right)=-2 a \frac{d e_{\theta}}{d z^{\prime}}
$$

The term involving $e_{\theta}\left(z^{\prime}\right)$ in Eq. (4.8) is therefore of order $1 /(k a)$ compared with the term in $e_{z}\left(z^{\prime}\right)$ and can, consequently, be neglected. The solution for $e_{z}\left(z^{\prime}\right)$ is then [6]

$$
e_{z}\left(z^{\prime}\right) \cong-\frac{(1-j)}{2 \sqrt{\pi k z^{\prime}}} .
$$

In order to obtain the impedance, defined in Eq. (2.3), we use Eq. (2.7) to show that

$$
\left.\frac{\partial E_{z}}{\partial x}\right|_{r=0}=\int_{-\infty}^{\infty} \frac{\kappa}{2} \frac{d q A(q)}{J_{1}(\kappa a)} e^{-j q z} .
$$

Equation (2.3) then becomes

$$
Z_{x}(k)=-\frac{q}{Q \Delta_{x} k} \int_{-\infty}^{\infty} \frac{\kappa d q A(q)}{2 J_{1}(\kappa a)} \int_{-\infty}^{\infty} d z e^{j(k-q) z} .
$$

The integral over $z$ in Eq. (4.13) is $2 \pi \delta(q-k)$, leading to

$$
Z_{x}(k)=-\frac{2 \pi A(k)}{Q \Delta_{x} k a}=-\frac{1}{Q \Delta_{x} k a} \int_{0}^{g} d z E_{z}(z) e^{j k z} .
$$

Using Eq. (2.17), we find that

$$
\frac{Z_{x}(k)}{Z_{0}}=-\frac{1}{\pi k a^{3}} \int_{0}^{g} d z^{\prime} e_{z}\left(z^{\prime}\right) \cong \frac{(1-j)}{a^{3}} \sqrt{\frac{g}{\pi^{3} k^{3}}} .
$$

We note that the high frequency dependence of the transverse impedance for a single cavity arises essentially from the TM cavity and pipe kernels. Furthermore, we see that

$$
\frac{Z_{x}(k)}{Z_{0}} \cong \frac{2}{k a^{2}} \frac{Z_{\|}(k)}{Z_{0}},
$$

where $Z_{\|}(k)$ is the high frequency limit for the longitudinal impedance [1], a result which also applies to a lossy beam pipe at any frequency [7].

In fact, in the high-frequency limit for a single cavity, simple arguments of diffraction theory can be used to obtain the same factor $2 /\left(k a^{2}\right)$ as in Eq. (4.16) [8]. Here we used a more rigorous treatment and showed that TE modes give only next-order frequency corrections, ensuring that only TM modes will govern the high-frequency behavior of the transverse impedance of a single cavity and providing the factor $2 /\left(k a^{2}\right)$.

\section{PERIODIC PILLBOX CAVITIES}

We are now ready to extend the calculation of the transverse impedance to a periodic array of identical cavities. The geometry of the system is shown in Fig. 2, with $g$ being the length of each cavity and $L$ being the axial distance (center to center) between two adjacent cavities.

We obtain the integral equations satisfied by the fields using the same steps as we followed for the case of a single cavity. The only difference is that in doing the field matching in each cavity one has to take into account the contributions to the fields coming from all the other cavities. For simplicity we first outline the calculation for the case in which only the TM modes are present. In this case [see Eqs. (2.7) and (2.12)], the tangential electric field in the $z$ direction at $r=a$ is

$$
E_{z}(z)=\int_{-\infty}^{\infty} d q A(q) e^{-j q z}
$$

and the corresponding magnetic field in the $\theta$ direction is

$$
Z_{0} H_{\theta}(z)=-j \int_{-\infty}^{\infty} d q A(q) \mu(q) e^{-j q z}+\frac{Q \Delta_{x} Z_{0}}{\pi a^{2}} e^{-j k z},
$$

where $\mu(q)=k J_{1}^{\prime}(\kappa a) /\left[J_{1}(\kappa a) \kappa\right]$. The component of the magnetic field in the $m$ th cavity can then be written as

$$
\begin{aligned}
Z_{0} H_{\theta}^{(m)}(t) \equiv & Z_{0} H_{\theta}(z=t+m L) \\
= & -j \int_{-\infty}^{\infty} d q A(q) \mu(q) e^{-j q(t+m L)} \\
& +\frac{Q \Delta_{x} Z_{0}}{\pi a^{2}} e^{-j k(t+m L)}
\end{aligned}
$$

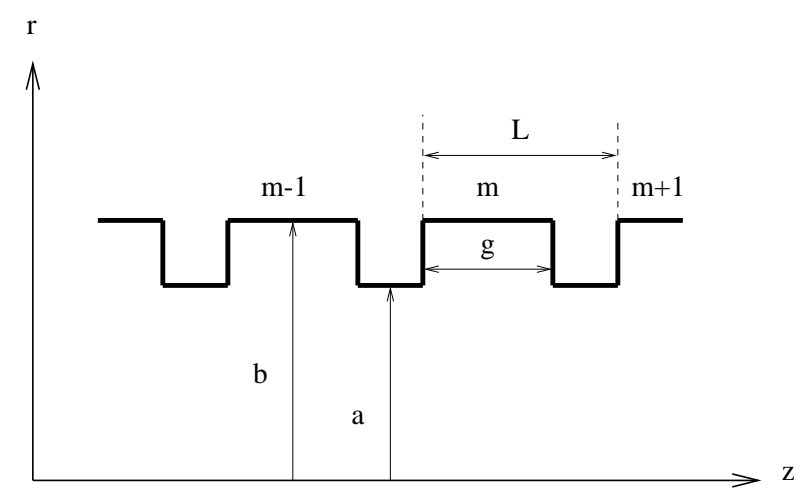

FIG. 2. The layout of geometry for a periodic array of cavities. 
where the function $A(q)$ is obtained by inversion of Eq. (5.1) and can be expressed in terms of the $z$ component of the electric field in the $\ell$ th cell $E_{z}^{(\ell)}(t) \equiv E_{z}(z=$ $t+\ell L)$

$$
\begin{aligned}
A(q) & =\frac{1}{2 \pi} \int_{-\infty}^{\infty} d z E_{z}(z) e^{j q z} \\
& =\frac{1}{2 \pi} \sum_{\ell=-\infty}^{\infty} e^{j q \ell L} \int_{0}^{g} d t E_{z}^{(\ell)}(t) e^{j q t} .
\end{aligned}
$$

Substitution of Eq. (5.4) into Eq. (5.3) leads to

$$
\begin{aligned}
Z_{0} H_{\theta}^{(m)}(t)= & -\sum_{\ell} \int_{0}^{g} d t^{\prime} P_{m, \ell}\left(t-t^{\prime}\right) E_{z}^{(\ell)}\left(t^{\prime}\right) \\
& +\frac{Q \Delta_{x} Z_{0}}{\pi a^{2}} e^{-j k(t+m L)},
\end{aligned}
$$

where

$$
P_{m, \ell}(u)=P_{m-\ell}(u) \equiv \frac{j}{2 \pi} \int_{-\infty}^{\infty} d q \mu(q) e^{-j q(m-\ell) L} e^{-j q u} .
$$

We now make the substitutions corresponding to Eqs. (2.15)-(2.18), recognizing that the fields stimulated by the traveling charge acquire a phase advance of $\exp (-j k L)$ from one cavity to the next. Thus we write

$$
\begin{gathered}
E_{z}^{(\ell)}\left(t^{\prime}\right) \equiv \frac{Q \Delta_{x} Z_{0}}{\pi a^{2}} e^{-j k\left(t^{\prime}+\ell L\right)} e\left(t^{\prime}\right), \\
Z_{0} H_{\theta}^{(m)}(t) \equiv \frac{Q \Delta_{x} Z_{0}}{\pi a^{2}} e^{-j k(t+m L)} h(t),
\end{gathered}
$$

and obtain

$$
h(t)=-\int_{0}^{g} d z^{\prime} \mathcal{P}\left(t-t^{\prime}\right) e\left(t^{\prime}\right)+1
$$

with

$$
\mathcal{P}(u)=\frac{j}{2 \pi} \sum_{\ell=-\infty}^{\infty} \int_{-\infty}^{\infty} d q \mu(q) e^{j(k-q)(u+(m-\ell) L)} .
$$

The integral over $q$ goes, as earlier, below all poles on the negative $q$ axis and above those on the positive axis. For positive values of $u+(m-\ell) L$ we close the contour in the lower half-plane, while for negative values of $u+(m-\ell) L$ we close the contour in the upper half- plane, thereby obtaining the exponential factor

$$
e^{j k(u+(m-\ell) L)-j\left(b_{s} / a\right)|u+(m-\ell) L|},
$$

where $b_{s}$ is the same as that defined in Eq. (A9). At high frequency we therefore have rapid phase oscillations and a vanishing kernel for $u+(m-\ell) L<0$. We obtain nonvanishing contributions only for $m=\ell$ and $u>0$, and for $m>\ell$ for all values of $u$. In fact, for $m=\ell$ these generate exactly our earlier pipe kernels. And we also obtain contributions for all $u$ as long as $\ell<m$, but none for $\ell>m$. The sum over $\ell$ in Eq. (5.10) can then be evaluated by taking the $\ell=m$ term from the single cavity pipe kernel and by writing

$$
\begin{aligned}
\sum_{\ell=-\infty}^{\ell=m-1} e^{j(k-q) L(m-\ell)} & =\sum_{p=1}^{\infty} e^{j(k-q) p L} \\
& =\frac{e^{j\left(k-b_{s} / a\right) L}}{1-e^{j\left(k-b_{s} / a\right) L}}
\end{aligned}
$$

for the pole at $q=b_{s} / a$, where we invoke a slightly lossy beam pipe wall to obtain convergence of the sum over $p$. The same reasoning outlined here can also be repeated for the field contributions coming from the TE modes. The only difference is that in the expression analogous to Eq. (5.12) one has to replace the roots $b_{s}$ of the Bessel function $J_{1}$ with the roots $b_{t}$ of the Bessel function $J_{1}^{\prime}$.

We now conclude that the pipe kernels in Eqs. (4.3) and (4.4) need to be augmented by a term proportional to the factors

$$
f_{s}=\frac{e^{j\left(k-b_{s} / a\right) L}}{1-e^{j\left(k-b_{s} / a\right) L}} \quad \text { and } \quad f_{t}=\frac{e^{j\left(k-b_{t} / a\right) L}}{1-e^{j\left(k-b_{t} / a\right) L}},
$$

respectively. Specifically,

$$
\begin{aligned}
& \mathcal{P}^{p}(u) \rightarrow \mathcal{P}^{p}(u)+\mathcal{P}^{L}(u), \\
& \mathcal{Q}^{p}(u) \rightarrow \mathcal{Q}^{p}(u)+\mathcal{Q}^{L}(u),
\end{aligned}
$$

where $\mathcal{P}^{L}(u)$ and $\mathcal{Q}^{L}(u)$ are obtained by multiplying the summands in the kernels in Eqs. (A14)-(A16) by $f_{s}$ and $f_{t}$, as appropriate. Futhermore, the forms to be used for Eqs. (A14)-(A16) are the ones used earlier for $u>0$ only, since $(m-\ell) L+u>0$ even for $u<0$. Thus we have

$$
\begin{gathered}
\mathcal{P}_{11}^{L}(u)+\mathcal{Q}_{11}^{L}(u) \cong-k \sum_{s=1}^{\infty} \frac{e^{j\left(k-b_{s} / a\right)(u+L)}}{b_{s}\left[1-e^{j\left(k-b_{s} / a\right) L}\right]}-\frac{1}{k a^{2}} \sum_{t=1}^{\infty} \frac{b_{t} e^{j\left(k-b_{t} / a\right)(u+L)}}{\left(p_{t}^{2}-1\right)\left[1-e^{j\left(k-b_{t} / a\right) L}\right]}, \\
\mathcal{Q}_{12}^{L}(u)=\mathcal{Q}_{21}^{L}(u) \cong-\frac{j}{k a^{2}} \sum_{t=1}^{\infty} \frac{p_{t}^{2} e^{j\left(k-b_{t} / a\right)(u+L)}}{\left(p_{t}^{2}-1\right)\left[1-e^{j\left(k-b_{t} / a\right) L}\right]}, \\
\tilde{Q}_{22}^{L}(u) \cong-\frac{j}{k a} \sum_{t=1}^{\infty} \frac{p_{t}^{2}\left(k a+b_{t}\right) e^{j\left(k-b_{t} / a\right)(u+L)}}{\left(p_{t}^{2}-1\right) b_{t}\left[1-e^{j\left(k-b_{t} / a\right) L}\right]} .
\end{gathered}
$$

And for high frequency we use $k-b_{s} / a \cong p_{s}^{2} / 2 k a^{2}, k-b_{t} / a \cong p_{t}^{2} / 2 k a^{2}$ to obtain [9] 


$$
\begin{aligned}
\mathcal{P}_{11}^{L}(u)+\mathcal{Q}_{11}^{L}(u) & \cong \frac{1}{a} \sum_{s=1}^{\infty} \frac{e^{j p_{s}^{2} u / 2 k a^{2}}}{1-e^{-j p_{s}^{2} L / 2 k a^{2}}}+\frac{1}{a} \sum_{t=1}^{\infty} \frac{e^{j p_{t}^{2} u / 2 k a^{2}}}{\left(p_{t}^{2}-1\right)\left[1-e^{-j p p_{t}^{2} L / 2 k a^{2}}\right]}, \\
\mathcal{Q}_{12}^{L}(u)= & \mathcal{Q}_{12}^{L}(u) \cong \frac{j}{k a^{2}} \sum_{t=1}^{\infty} \frac{p_{t}^{2}}{p_{t}^{2}-1} \frac{e^{j p_{t}^{2} u / 2 k a^{2}}}{\left[1-e^{\left.-j p_{t}^{2} L / 2 k a^{2}\right]}\right.}, \\
& \tilde{Q}_{22}^{L}(u) \cong 2 j \sum_{t=1}^{\infty} \frac{p_{t}^{2}}{p_{t}^{2}-1} \frac{e^{j p_{t}^{2} u / 2 k a^{2}}}{\left[1-e^{-j p_{t}^{2} L / 2 k a^{2}}\right]} .
\end{aligned}
$$

Evaluation of these periodic kernels, and their role in the integral equations, continues in the next section. Incidentally, notice that the kernels associated with the cavity region (i.e., $\mathcal{P}^{c}$ and $\mathcal{Q}^{c}$ ) are the same as in a single cavity system [see Eqs. (3.18)-(3.22)].

\section{INTEGRAL EQUATIONS FOR PERIODIC CAVITIES}

We first evaluate the periodic kernels in Eqs. (5.18)-(5.20) for $g \ll L$, for which $|u| \ll L$. As a result, at high frequency

$$
\mathcal{P}_{11}^{L}(u)+\mathcal{Q}_{11}^{L}(u)=\frac{1}{a} \sum_{s=1}^{\infty} \frac{e^{j p_{s}^{2} u / 2 k a^{2}}}{1-e^{-j p_{s}^{2} L / 2 k a^{2}}}+\frac{1}{a} \sum_{t=1}^{\infty} \frac{e^{j p_{t}^{2} u / 2 k a^{2}}}{\left(p_{t}^{2}-1\right)\left(1-e^{-j p_{t}^{2} L / 2 k a^{2}}\right)} .
$$

For small $|u| / L$ the first approximation to Eq. (6.1) for large $k$ is

$$
\mathcal{P}_{11}^{L}(u)+\mathcal{Q}_{11}^{L}(u) \cong-\frac{2 j k a}{L}\left[\sum_{s=1}^{\infty} \frac{1}{p_{s}^{2}}+\sum_{t=1}^{\infty} \frac{1}{p_{t}^{2}\left(p_{t}^{2}-1\right)}\right] .
$$

We now use Eq. (A1) for small $z$ to show that

$$
\sum_{s=1}^{\infty} \frac{1}{p_{s}^{2}}=\frac{1}{8}
$$

We also expand Eq. (A2) for small $z$ to obtain

$$
\sum_{t=1}^{\infty} \frac{1}{p_{t}^{2}}=\frac{3}{8} \text {. }
$$

Using Eq. (A5), we then have

$$
\sum_{t=1}^{\infty} \frac{1}{p_{t}^{2}\left(p_{t}^{2}-1\right)}=\frac{1}{8}
$$

so that Eq. (6.2) becomes

$$
\mathcal{P}_{11}^{L}(u)+\mathcal{Q}_{11}^{L}(u) \cong-\frac{j k a}{2 L} .
$$

Similarly,

$$
\mathcal{Q}_{12}^{L}(u)=\mathcal{Q}_{21}^{L}(u) \cong \frac{2}{L} \sum_{t=1}^{\infty} \frac{1}{p_{t}^{2}-1}=\frac{1}{L},
$$

and

$$
\tilde{Q}_{22}^{L}(u) \cong \frac{4 a}{L} \sum_{t=1}^{\infty} \frac{1}{p_{t}^{2}-1}=\frac{2 a}{L} .
$$

Comparison with Eqs. (4.5)-(4.7) shows that in each case the periodic kernels are larger than the pipe and cavity kernels by a factor of order $(k g)^{1 / 2}(a / L)$. Returning to Eqs. (4.8) and (4.9), we again see that, for large $k, e_{z}\left(z^{\prime}\right)$ and $a_{d e}\left(z^{\prime}\right) / d z^{\prime}$ are comparable in magnitude and that the $e_{\theta}$ term in Eq. (4.8) is again of order $1 /(\mathrm{ka})$ compared with the $e_{z}$ term. We therefore neglect the $e_{\theta}$ term in
Eq. (4.1), as well as all other terms of order $1 / k$ to obtain

$$
\int_{0}^{g} d z^{\prime}\left[\mathcal{P}_{11}(u)+\mathcal{P}_{11}^{L}(u)+\mathcal{Q}_{11}^{L}(u)\right] e_{z}\left(z^{\prime}\right)=1,
$$

where the single cavity kernel, $\mathcal{P}_{11}(u)$, given in Eq. (4.5), is

$$
\mathcal{P}_{11}(u)= \begin{cases}0, & u<0 \\ -(1-j) \sqrt{k / \pi u}, & u>0\end{cases}
$$

As for the case of a single cavity, we find that in the high frequency limit we can reduce the original system of two integral equations to a single equation for the unknown axial component of the electric field $e_{z}$. This integral equation has the same form as the one in the calculation of the longitudinal impedance [2]. Therefore, for the transverse impedance of a periodic structure at high frequency we finally obtain

$$
Z_{x}(g / L)=\frac{2}{k a^{2}} Z_{\|}(g / L) .
$$

The problem is then reduced to calculating $Z_{\|}$from the solution of the integral equation for $e_{z}$.

We have earlier [2] obtained the longitudinal impedance for periodic cavities in the limit $g \ll L$. It has been pointed out by Yokoya [10], Stupakov [11], and Bane et al. [12] that these results are in slight disagreement with the results for larger $g / L$. This is indeed the case because when the condition $g \ll L$ is not valid, a more accurate treatment of [2] with an additional frequency correction term is needed, as was pointed out by Yokoya [10]. In fact, for a linac consisting of a 
pipe loaded with thin irises, one is interested in the limit $g / L \rightarrow 1$.

In the limit $g / L=1$ in the high-frequency region, an infinite periodic array of cavities can be approximated by an infinite periodic array of perfectly conducting halfplanes. The problem of diffraction of a plane wave by an infinite array of periodic half-planes can be solved analytically, as was shown by Vainshtein [13]. Stupakov used Vainshtein's theory to obtain an analytic result for the impedance for the case $g / L=1[11,14]$. However,

in a real iris loaded structure $g / L$ is not exactly unity; thus, it would be highly desirable to have an analytic approximation valid for any $g / L$. Yokoya showed that it was necessary to keep an extra term of order $k^{-1 / 2}$ compared with the coupling kernel, given by Eq. (6.2), in order to have a result valid for general $g / L[10]$. He then solved the integral equation for the longitudinal impedance numerically, and confirmed Stupakov's result in the limit $g / L=1$ to very good accuracy.

We now repeat Yokoya's derivation for general $g / L$ by first rewriting Eq. (6.1) as

$$
\mathcal{P}_{11}^{L}(u)+\mathcal{Q}_{11}^{L}(u)=-\frac{j k a}{2 L}+\frac{1}{a} \sum_{s=1}^{\infty}\left[\frac{e^{j p_{s}^{2} u / 2 k a^{2}}}{1-e^{-j p_{s}^{2} L / 2 k a^{2}}}+\frac{2 j k a^{2}}{L p_{s}^{2}}\right]+\frac{1}{a} \sum_{t=1}^{\infty} \frac{1}{p_{t}^{2}-1}\left[\frac{e^{j p_{t}^{2} u / 2 k a^{2}}}{1-e^{-j p_{t}^{2} L / 2 k a^{2}}}+\frac{2 j k a^{2}}{L p_{t}^{2}}\right]
$$

Since the first bracket [] vanishes for large $k$ unless $p_{s}^{2}$ is of order $k a^{2} / L$, we can convert it to an integral. Specifically, we write

$$
w=\frac{1-j}{2} \frac{p_{s}}{a} \sqrt{\frac{L}{k}}, \quad \sum_{s=1}^{\infty} \Rightarrow(1+j) \frac{a}{\pi} \sqrt{\frac{k}{L}} \int_{0}^{\infty} d w .
$$

But the summand in the second bracket [] has an additional factor $\left(p_{t}^{2}-1\right)^{-1}$ which, for $p_{t}^{2} \sim k a^{2} / L$ reduces the contribution to Eq. (6.12) by a factor of order $1 / k$, and can be neglected in Eq. (6.12). Thus we have

$$
\mathcal{P}_{11}^{L}(u)+\mathcal{Q}_{11}^{L}(u)=-\frac{j k a}{2 L}-\frac{(1+j)}{\pi} \sqrt{\frac{k}{L}} \int_{0}^{\infty} d w\left[\frac{e^{-u w^{2} / L}}{e^{w^{2}}-1}-\frac{1}{w^{2}}\right] .
$$

The second term on the right-hand side of Eq. (6.14) is the anticipated correction term of order $k^{-1 / 2}$. The resulting integral equation can be approximately solved (see Appendix D) with the following result for the impedance per period $Z_{\|}(g / L)$ :

$$
\frac{Z_{\|}}{Z_{0}}=\frac{-j L}{\pi k a^{2}}\left[1+(1-j) \frac{L}{a} \sqrt{\frac{\pi}{k g}} \lambda(g / L)\right]^{-1}
$$

with

$$
\begin{aligned}
\lambda(\xi) \cong & 1+\frac{\alpha_{0}}{\pi} \xi^{1 / 2}-\frac{\alpha_{1}}{3 \pi} \xi^{3 / 2}+\frac{13 \alpha_{2}}{45 \pi} \xi^{5 / 2} \\
& +\frac{8 \alpha_{1}^{2}}{45 \pi^{2}} \xi^{3}-O\left(\xi^{7 / 2}\right)
\end{aligned}
$$

In the limit $g \ll L$ we have $\lambda=1$, and one recovers our earlier expression for the longitudinal impedance [2]. For $g=L$, Stupakov obtains $\lambda(1)=-\zeta(1 / 2) / \pi \cong$ 0.464845 [11], in agreement with Yokoya's numerical result [10], where $\zeta$ denotes the Riemann zeta function.

Equations (6.11) and (6.15) provide an analytic description of the transverse and longitudinal impedance at high frequency for an infinite array of periodic cavities. These expressions are valid when $N L \gg k a^{2}$, where $N$ is the number of cavities. For $k a^{2} / L \geq N \gg 1$ there is a transition to the result valid for a finite number of cavities, and one needs to use a different expression for the longitudinal impedance $[14,15]$.

\section{RELATION BETWEEN TRANSVERSE AND LONGITUDINAL IMPEDANCE}

In earlier sections we obtained expressions for the smoothed high frequency transverse coupling impedance for both a single azimuthally symmetric cavity and a periodic array of such cavities. What we found in both cases is the result in Eqs. (4.16) and (6.11), namely,

$$
Z_{x}(k) \cong \frac{2}{k a^{2}} Z_{\|}(k),
$$

for all values of $g / L$, as long as $k g \gg 1, k a \gg 1$.

In the course of our detailed derivation we needed a second term in the expansion of the coupling kernel, $\mathcal{P}_{11}^{L}(u)+\mathcal{Q}_{11}^{L}(u)$, which was of order $k^{-1 / 2}$ compared with the first term, as displayed in Eq. (6.14). As pointed out by Yokoya [10], this second term was required for nonvanishing values of $g / L$.

The validity of the factor $2 /\left(k a^{2}\right)$ constitutes the main result of this paper. It allows one to make a simple estimate for the smoothed transverse coupling impedance at high frequency. We remind the reader that, because the detailed dependence at high frequency involves many contributions from closely spaced cutoff frequencies, the behavior is governed by the smoothed version of the impedance [14]. This is consistent with the presence of the factor $2 /\left(k a^{2}\right)$ for a beam pipe with finite resistivity [14], as well as for a corrugated pipe over a wide range of frequency $[16,17]$. Also, for a small azimuthally symmetric pillbox, relation between the electric and magnetic fields which is valid for 
a resistive wall can be given in terms of the "effective" impedance $[14,18]$ which could again justify the use of the factor $2 /\left(k a^{2}\right)$. Kurennoy suggests that this result may apply more generally to structures with azimuthal symmetry [19]. However, the range of applicability of this relation even for the azimuthally symmetric structures requires further investigation.

\section{SUMMARY}

In this paper we address the question of the transverse coupling impedance for a periodic array of cavities at high frequency. We first derive a result for the transverse impedance of a single obstacle at high frequency, and then proceed to the periodic case. The problem is more complicated than for the longitudinal impedance, since both TM and TE waveguide modes are present, which requires matching two components of the magnetic field at the boundary between the waveguide and the obstacle. We show that, in the high frequency limit, the TM contribution dominates for a single obstacle, while the TE contribution must be included in the periodic case. Nevertheless, we find the same relation between the longitudinal and transverse impedances as exists for the resistive wall impedance of a beam pipe. The validity of the factor $2 /\left(k a^{2}\right)$ between the longitudinal and transverse impedances allows simple estimates of the transverse impedance at high frequency.

\section{ACKNOWLEDGMENTS}

We wish to acknowledge the support of the U.S. Department of Energy. We are grateful to K. Yokoya for sharing his notes with us and for making several useful comments. We also wish to thank S. Kurennoy for helpful comments.

\section{APPENDIX A}

In order to evaluate the pipe kernels in Eqs. (2.21)(2.24) we need formulas that express the ratios of Bessel functions in terms of the zeros of the denominator. The first two formulas can be easily established by using the property that two analytic functions are identical if they have the same Laurent series. Specifically, we write

$$
\frac{J_{1}^{\prime}(z)}{z J_{1}(z)}=\frac{1}{z^{2}}+2 \sum_{s=1}^{\infty} \frac{1}{z^{2}-p_{s}^{2}}
$$

where $J_{1}\left(p_{s}\right)=0$, and

$$
\frac{J_{1}^{\prime \prime}(z)}{z J_{1}^{\prime}(z)}=2 \sum_{t=1}^{\infty} \frac{1}{z^{2}-p_{t}^{2}}
$$

where $J_{1}^{\prime}\left(p_{t}\right)=0$. To derive the third formula we first write the differential equation satisfied by $J_{1}(z)$,

$$
\frac{J_{1}^{\prime \prime}(z)}{z J_{1}^{\prime}(z)}+\frac{1}{z^{2}}+\frac{J_{1}(z)}{z J_{1}^{\prime}(z)}\left(1-\frac{1}{z^{2}}\right)=0
$$
and obtain

$$
\begin{aligned}
\frac{J_{1}(z)}{z J_{1}^{\prime}(z)} & =-\frac{1}{z^{2}-1}-2 \frac{z^{2}}{z^{2}-1} \sum_{t=1}^{\infty} \frac{1}{z^{2}-p_{t}^{2}} \\
& =-\frac{1}{z^{2}-1}+\frac{2}{z^{2}-1} \sum_{t=1}^{\infty} \frac{1}{p_{t}^{2}-1}-2 \sum_{t=1}^{\infty} \frac{p_{t}^{2}}{\left(p_{t}^{2}-1\right)\left(z^{2}-p_{t}^{2}\right)} .
\end{aligned}
$$

Equation (A4) can be simplified by using Eqs. (A3) and (A2) to obtain

$$
\frac{J_{1}^{\prime \prime}(1)}{J_{1}^{\prime}(1)}=-1=2 \sum_{t=1}^{\infty} \frac{1}{1-p_{t}^{2}},
$$

leading to

$$
\frac{J_{1}(z)}{z J_{1}^{\prime}(z)}=2 \sum_{t=1}^{\infty} \frac{p_{t}^{2}}{\left(p_{t}^{2}-1\right)\left(p_{t}^{2}-z^{2}\right)}
$$

We now proceed to calculate the pipe kernels, using Eqs. (A1) and (A6). Specifically, we set

$$
z^{2}=\kappa^{2} a^{2}=k^{2} a^{2}-q^{2} a^{2}
$$

leading to

$$
\mathcal{P}_{11}^{p}(u)=-\frac{j k}{2 \pi a} e^{j k u} \int_{-\infty}^{\infty} d q e^{-j q u}\left[\frac{1}{\left(q^{2}-k^{2}\right)}+2 \sum_{s=1}^{\infty} \frac{1}{q^{2}-b_{s}^{2} / a^{2}}\right]
$$

where

$$
b_{s}=\left(k^{2} a^{2}-p_{s}^{2}\right)^{1 / 2}=-j\left(p_{s}^{2}-k^{2} a^{2}\right)^{1 / 2},
$$


and where the contour goes below the poles at $q=-k,-b_{s} / a$ and above the poles at $q=k, b_{s} / a$. Thus

$$
\mathcal{P}_{11}^{p}(u)=-\frac{e^{-j k|u|+j k u}}{2 a}-k \sum_{s=1}^{\infty} \frac{e^{-j b_{s}|u| / a+j k u}}{b_{s}} .
$$

In order to evaluate $\mathcal{Q}_{11}^{p}(u)$, we write

$$
\frac{q^{2}}{\kappa^{3} a} \frac{J_{1}(\kappa a)}{J_{1}^{\prime}(\kappa a)}=\left[\frac{k^{2} a^{2}}{z^{3}} \frac{J_{1}(z)}{J_{1}^{\prime}(z)}-\frac{1}{z} \frac{J_{1}(z)}{J_{1}^{\prime}(z)}\right] .
$$

From Eq. (A6) we have

$$
\begin{aligned}
\frac{J_{1}(z)}{z^{3} J_{1}^{\prime}(z)} & =2 \sum_{t=1}^{\infty} \frac{p_{t}^{2}}{\left(p_{t}^{2}-1\right) z^{2}\left(p_{t}^{2}-z^{2}\right)} \\
& =\frac{2}{z^{2}} \sum_{t=1}^{\infty} \frac{1}{\left(p_{t}^{2}-1\right)}+2 \sum_{t=1}^{\infty} \frac{1}{\left(p_{t}^{2}-1\right)\left(p_{t}^{2}-z^{2}\right)}=\frac{1}{z^{2}}+2 \sum_{t=1}^{\infty} \frac{1}{\left(p_{t}^{2}-1\right)\left(p_{t}^{2}-z^{2}\right)} .
\end{aligned}
$$

Thus

$$
\mathcal{Q}_{11}^{p}(u)=\frac{e^{-j k|u|+j k u}}{2 a}-\frac{1}{k a^{2}} \sum_{t=1}^{\infty} \frac{b_{t}}{p_{t}^{2}-1} e^{-j b_{t}|u| / a+j k u} .
$$

We now combine Eqs. (A10) and (A11) to obtain

$$
\mathcal{P}_{11}^{p}(u)+\mathcal{Q}_{11}^{p}(u)=-k \sum_{-\infty}^{\infty} \frac{e^{j\left(k u-b_{s}|u| / a\right)}}{b_{s}}-\frac{1}{k a^{2}} \sum_{t=1}^{\infty} \frac{b_{t} e^{j\left(k u-b_{t}|u| / a\right)}}{p_{t}^{2}-1} .
$$

Similarly,

$$
\begin{gathered}
\mathcal{Q}_{12}^{p}(u)=\mathcal{Q}_{21}^{p}(u)=\mp \frac{j}{k a} \sum_{t=1}^{\infty} \frac{p_{t}^{2}}{\left(p_{t}^{2}-1\right)} e^{-j b_{t}|u| / a+j k u}, \\
\tilde{Q}_{22}^{p}(u)=-\frac{j}{k a} \sum_{t=1}^{\infty} \frac{p_{t}^{2}\left(k a \pm b_{t}\right)}{\left(p_{t}^{2}-1\right) b_{t}} e^{-j b_{t}|u| / a+j k u},
\end{gathered}
$$

where the upper and lower signs correspond to $u>0$ and $u<0$ respectively, with

$$
b_{t}=\left(k^{2} a^{2}-p_{t}^{2}\right)^{1 / 2}=-j\left(p_{t}^{2}-k^{2} a^{2}\right)^{1 / 2} .
$$

\section{APPENDIX B}

We obtain the high frequency smoothed pipe kernels in the following way. Both terms in Eq. (A14) oscillate rapidly for $u<0$, averaging to zero. But for $u>0$, the oscillations no longer disappear when $p_{s} \ll k a, p_{t} \ll$ $k a$. Thus we expand for $p_{s} \ll k a, p_{t} \ll k a$, writing

$$
k-b_{s} / a \cong p_{s}^{2} / 2 k a^{2}, \quad k-b_{t} / a \cong p_{t}^{2} / 2 k a^{2},
$$

and obtain for the smoothed high frequency kernel

$$
\begin{aligned}
\mathcal{P}_{11}^{p}(u)+\mathcal{Q}_{11}^{p}(u) \cong & -\frac{1}{a} \sum_{s=1}^{\infty} e^{j p_{s}^{2} u / 2 k a^{2}} \\
& -\frac{1}{a} \sum_{t=1}^{\infty} \frac{e^{j p_{t}^{2} u / 2 k a^{2}}}{p_{t}^{2}-1} .
\end{aligned}
$$

For high frequency the sum over $s$ can be converted to an integral over $\int d p_{s} / \pi$, while the sum over $t$ can be performed without the help of the exponential, using Eq. (A5), with the result

$$
\mathcal{P}_{11}^{p}(u)+\mathcal{Q}_{11}^{p}(u)= \begin{cases}0, & u<0, \\ -(1-j) \sqrt{k /(4 \pi u)}, & u>0,\end{cases}
$$

where we retain only the leading term for large $k$. In fact, for large $k$, the results in Eq. (B3) come only from $\mathcal{P}_{11}^{p}(u)$ for a single obstacle.

The sum over $t$ in Eq. (A15) is controlled by the behavior for large $t$, where $p_{t}^{2} /\left(p_{t}^{2}-1\right) \cong 1$. As a result, the dominant behavior for large $k$ is

$$
\mathcal{Q}_{12}^{p}(u)=\mathcal{Q}_{21}^{p}(u) \cong \begin{cases}0, & u<0, \\ (1-j) / \sqrt{4 \pi k u a^{2}}, & u>0 .\end{cases}
$$

Similarly, the dominant values for $p_{t}$ in Eq. (A16) are of order $\left(k a^{2} / u\right)^{1 / 2}$, so that the leading term for large $k$ is

$$
\tilde{Q}_{22}^{p}(u) \cong \begin{cases}0, & u<0, \\ (1-j) / \sqrt{\pi k u}, & u>0 .\end{cases}
$$

The results in Eqs. (B3)-(B5) represent the dominant behavior, after smoothing, for large $k$.

\section{APPENDIX C}

As we examine the cavity kernels in Eqs. (3.18)(3.22), we see that all terms will oscillate rapidly for large $k$ unless $n \pi / g$ is near $k$. Thus, for the smoothed behavior 
for large $k$, the sums will be dominated by these large values of $n$, and we will have

$$
\mathcal{P}_{11}^{c}\left(z, z^{\prime}\right) \cong \mathcal{P}_{11}^{c}(u) \cong-\frac{j k a}{2 g} \sum_{n} e^{j(k-n \pi / g) u} \frac{F^{\prime}(\sigma a)}{\sigma a F(\sigma a)}
$$

$$
\begin{aligned}
\mathcal{Q}_{11}^{c}\left(z, z^{\prime}\right) & \cong \mathcal{Q}_{11}^{c}(u) \\
& \cong \frac{j k a}{2 g} \sum_{n} e^{j(k-n \pi / g) u} \frac{G(\sigma a)}{(\sigma a)^{3} G^{\prime}(\sigma a)},
\end{aligned}
$$

$$
\begin{aligned}
\mathcal{Q}_{12}^{c}\left(z, z^{\prime}\right) & \cong \mathcal{Q}_{12}^{c}(u) \cong \mathcal{Q}_{21}^{c}(u) \\
& \cong-\frac{1}{2 g} \sum_{n} e^{j(k-n \pi / g) u} \frac{G(\sigma a)}{\sigma a G^{\prime}(\sigma a)},
\end{aligned}
$$

$$
\begin{aligned}
\mathcal{Q}_{22}^{c}\left(z, z^{\prime}\right) & \cong \mathcal{Q}_{22}^{c}(u) \\
& \cong-\frac{j}{2 k g a} \sum_{n} e^{j(k-n \pi / g) u} \frac{\sigma a G(\sigma a)}{G^{\prime}(\sigma a)},
\end{aligned}
$$

where

$$
\sigma^{2} a^{2}=k^{2} a^{2}-(n \pi a / g)^{2} \cong 2 k a^{2}(k-n \pi / g) .
$$

The main contributions to the sum over $n$ occur for $k-$ $n \pi / g \sim \mathcal{O}(1 / u) \sim \mathcal{O}(1 / g)$. Thus we must evaluate $F(\sigma a), F^{\prime}(\sigma a), G(\sigma a)$, and $G^{\prime}(\sigma a)$ for large $\sigma a$.

We first consider

$$
\frac{F^{\prime}(\sigma a)}{F(\sigma a)}=\frac{H_{+}^{\prime}(\sigma a)-\lambda H_{-}^{\prime}(\sigma a)}{H_{+}(\sigma a)-\lambda H_{-}(\sigma a)} .
$$

Let us examine the wall impedance for the TM modes at $r=b$. We form, using Eqs. (3.1) and (3.11),

$$
\frac{E_{z}(b, z)}{Z_{0} H_{\theta}(b, z)}=\frac{j \sigma F(\sigma b)}{k F^{\prime}(\sigma b)},
$$

and now require that

$$
\frac{E_{z}}{Z_{0} H_{\theta}} \cong-\frac{(1+j)}{2} k \delta \equiv \frac{Z_{w}}{Z_{0}}
$$

where $\delta$ is the (extremely small) skin depth at frequency $k c / 2 \pi$ and $Z_{w}$ is the wall impedance. Thus we have

$$
\frac{F(\sigma b)}{F^{\prime}(\sigma b)}=\frac{H_{+}(\sigma b)-\lambda H_{-}(\sigma b)}{H_{+}^{\prime}(\sigma b)-\lambda H_{-}^{\prime}(\sigma b)} \cong \frac{j-1}{2} \frac{k^{2} \delta}{\sigma} \text {. }
$$

We now use the asymptotic forms for $H_{ \pm}(\sigma b)$ and $H_{ \pm}^{\prime}(\sigma b)$ to obtain

$$
\frac{1-\lambda e^{-2 j \sigma b}}{1+\lambda e^{2 j \sigma b}} \cong-\frac{(1+j)}{2} \frac{k^{2} \delta}{\sigma}
$$

or

$$
\lambda \cong e^{2 j \sigma b}\left[1+(1+j) \frac{k^{2} \delta}{\sigma}\right]
$$

Substituting Eq. (C11) into Eq. (C6), and using the asymptotic forms for $H_{ \pm}(\sigma a), H_{ \pm}^{\prime}(\sigma a)$, we find

$$
\frac{F^{\prime}(\sigma a)}{F(\sigma a)} \cong j\left[\frac{1+e^{2 j(b-a) \sigma}(1+\epsilon)}{1-e^{2 j(b-a) \sigma}(1+\epsilon)}\right]
$$

where

$$
\epsilon=(1+j) k^{2} \delta / \sigma, \quad|\epsilon| \ll 1 .
$$

Because of Eq. (C5) and the exponential $e$, the righthand side of Eq. (C12) oscillates rapidly at high frequency. To obtain its average value, we average over a single oscillation by calculating

$$
\frac{F^{\prime}(\sigma a)}{F(\sigma a)} \cong \frac{j}{2 \pi} \int_{0}^{2 \pi} d \phi \frac{\left[1-\epsilon+e^{j \phi}\right]}{\left[1-\epsilon-e^{j \phi}\right]} .
$$

Equation (C14) is evaluated by the substitution $e^{j \phi}=$ $w$, leading to

$$
\frac{F^{\prime}(\sigma a)}{F(\sigma a)} \cong-\frac{1}{2 \pi} \oint \frac{d w}{w}\left(\frac{w+(1-\epsilon)}{w-(1-\epsilon)}\right),
$$

where the contour in the $w$ plane is the unit circle. But the integrand in Eq. (C15) has poles at $w=0$, and $w=1-\epsilon$, both of which are within the unit circle. Thus the contour in Eq. (C15) can be expanded to a circle of large radius, giving for the smoothed, high frequency value [20],

$$
\frac{F^{\prime}(\sigma a)}{F(\sigma a)} \cong-j
$$

A similar calculation for the TE modes leads to

$$
\frac{G(\sigma a)}{G^{\prime}(\sigma a)} \cong j
$$

The foregoing calculation is for $k>n \pi / g$. For $k<$ $n \pi / g$ we need to use the Bessel functions of imaginary argument to construct $F(u)$ and $G(u)$. In this case, for large $k$ we define

$$
\rho^{2} a^{2}=2 k a^{2}(n \pi / g-k)=-\sigma^{2} a^{2},
$$

and make the replacement

$$
\frac{F^{\prime}(\sigma a)}{\sigma a F(\sigma a)} \rightarrow-\frac{\tilde{F}^{\prime}(\rho a)}{\rho a \tilde{F}(\rho a)}
$$

where

$$
\tilde{F}(u)=K_{1}(u)-\lambda I_{1}(u) .
$$

There is no longer oscillation in the high frequency limit which becomes

$$
\frac{\tilde{F}^{\prime}(\rho a)}{\tilde{F}(\rho a)} \cong-1
$$

Similarly, with $\tilde{G}(u)=K_{1}(u)-\lambda I_{1}(u)$ we make the replacement

$$
\frac{G(\sigma a)}{\sigma a G^{\prime}(\sigma a)} \rightarrow \frac{\tilde{G}(\rho a)}{\rho a \tilde{G}^{\prime}(\rho a)},
$$


and obtain the limit

$$
\frac{\tilde{G}(\rho a)}{\tilde{G}^{\prime}(\rho a)} \cong-1 .
$$

We now complete the calculation of $\mathcal{P}_{11}^{c}(u)$ in Eq. (C1) by converting the sum over $n$ to an integral over $s \equiv n \pi / g-k$ from $s=-\infty$ to $+\infty$. The extension to $s=-\infty$ covers a range of rapid oscillation which does not change our result, and the conversion from a sum to an integral is equivalent to obtaining a smoothed local average over $k$. Thus we replace $F^{\prime}(\sigma a) /(\sigma a F(\sigma a))$ in Eq. (C1) by using Eqs. (C16), (C5), (C19), and (C21) to obtain

$$
\begin{aligned}
\frac{F^{\prime}(\sigma a)}{\sigma a F(\sigma a)} & \cong \frac{1}{\sqrt{2 k a^{2}}} \begin{cases}-j / \sqrt{-s}, & s<0 \\
1 / \sqrt{s}, & s>0\end{cases} \\
& =\frac{1}{\sqrt{2 k a}} \lim _{\epsilon \rightarrow 0}(s+j \epsilon)^{-1 / 2} .
\end{aligned}
$$

We then can write the cavity kernel in Eq. (C1) as

$$
\mathcal{P}_{11}^{c}(u) \cong-\frac{j \sqrt{k}}{2 \pi \sqrt{2}} \int_{-\infty}^{\infty} \frac{d s e^{-j s u}}{\sqrt{s}}
$$

where the branch point at $s=-j \epsilon$ has been moved to $s=0$, but the contour in Eq. (C25) is kept above the branch point.

Clearly, for $u<0$ the contour can be closed in the upper half-plane, enclosing no singularities, so that $\mathcal{P}_{11}^{c}(u)=0$ for $u<0$. For $u>0$ we distort the contour so that it travels from $s=-j \infty$ to $s=0$ (to the left of the branch cut) around the branch point at $s=0$ in a clockwise direction and returns from $s=0$ to $s=-j \infty$ (to the right of the branch cut). If we set $s=j t / u$ and reverse the direction of integration, we have

$$
\mathcal{P}_{11}^{c}(u)=\frac{j \sqrt{k}}{2 \pi \sqrt{2}} \frac{1+j}{\sqrt{2 u}} \int_{-\infty}^{0(+)} \frac{d t e^{t}}{t^{1 / 2}},
$$

where the contour is from $t=-\infty$ to $t=0$, around $t=0$ in a counterclockwise direction, and then back to $t=-\infty$. We now use the representation

$$
\frac{1}{\Gamma(p)}=\frac{1}{2 \pi j} \int_{-\infty}^{0(+)} \frac{d t e^{t}}{t^{p}}
$$

for the reciprocal of the gamma function (which is easily verifiable when $p$ is a positive integer and which represents an analytic continuation for all other values of $p)$. Thus we finally obtain

$$
\mathcal{P}_{11}^{c}(u)= \begin{cases}0, & u<0 \\ -\frac{1+j}{2} \sqrt{k /(\pi u)}, & u>0\end{cases}
$$

In a similar way we find, using Eqs. (C5), (C17), (C18), (C22), and (C23),

$$
\begin{aligned}
\frac{G(\sigma a)}{\sigma a G^{\prime}(\sigma a)} & \cong \frac{1}{\sqrt{2 k a^{2}}} \begin{cases}j / \sqrt{-s}, & s<0 \\
-1 / \sqrt{s}, & s>0\end{cases} \\
& =-\frac{1}{\sqrt{2 k a^{2} s}},
\end{aligned}
$$

where the branch point at $s=0$ lies below the contour from $s=-\infty$ to $+\infty$. The kernel $\mathcal{Q}_{11}^{c}(u)$ in Eq. (C2) can then be written as

$$
\mathcal{Q}_{11}^{c}(u) \cong \frac{j k a}{2 \pi} \int_{-\infty}^{\infty} \frac{d s e^{-j s u}}{\left(2 k a^{2} s\right)^{3 / 2}} .
$$

Clearly $\mathcal{Q}_{11}^{c}(u)$ vanishes for $u<0$. For $u>0$ we set $s=j t / u$ and reverse the direction of integration to find

$$
Q_{11}^{c}(u) \cong-\frac{\sqrt{j u}}{4 \pi a^{2} \sqrt{2 k}} \int_{-\infty}^{0(+)} \frac{d t e^{t}}{t^{3 / 2}},
$$

so that

$$
\mathcal{Q}_{11}^{c}(u)= \begin{cases}0, & u<0, \\ (1-j) \sqrt{u / k \pi} /\left(2 a^{2}\right), & u>0 .\end{cases}
$$

Comparison with Eq. (C28) shows that $\mathcal{Q}_{11}^{c}(u)$ is of order $\mathcal{P}_{11}^{c}(u) / k$ and can therefore be neglected, as was the situation in Eqs. (B2) and (B3) for $\mathcal{Q}_{11}^{p}(u)$.

For the kernel $\mathcal{Q}_{12}^{c}(u)=\mathcal{Q}_{21}^{c}(u)$ in Eq. (C3) using Eq. (C29), we find

$$
\mathcal{Q}_{12}^{c}(u)=Q_{21}^{c}(u)=\frac{1}{2 \pi} \int_{-\infty}^{\infty} \frac{d s e^{-j s u}}{\sqrt{2 k a^{2} s}} .
$$

Once again the kernel vanishes for $u<0$. For $u>0$, we set $s=j t / u$ and reverse the direction of integration to obtain

$$
\mathcal{Q}_{12}^{c}(u)=\mathcal{Q}_{21}^{c}(u)= \begin{cases}0, & u<0, \\ (1-j) / \sqrt{4 \pi k u a^{2}}, & u>0 .\end{cases}
$$

Finally, we need to obtain $\tilde{\mathcal{Q}}_{22}^{c}(u)$ which we define, consistent with the definition of $\tilde{Q}_{22}^{p}(u)$ in Eq. (2.25), as

$\tilde{\mathcal{Q}}_{22}^{c}(u)=-\int^{z^{\prime}} d z^{\prime} \mathcal{Q}_{22}^{c}(u)=\int^{u} d u \mathcal{Q}_{22}^{c}(u)$.

Using Eqs. (C4) and (C5), we find

$\tilde{\mathcal{Q}}_{22}^{c}(u) \cong-\frac{a}{g} \sum_{n} e^{j(k-n \pi / g) u} \frac{G(\sigma a)}{\sigma a G^{\prime}(\sigma a)}=2 a \mathcal{Q}_{12}^{c}(u)$.

Specifically,

$$
\tilde{Q}_{22}^{c}(u) \cong \begin{cases}0, & u<0, \\ (1-j) / \sqrt{\pi k u}, & u>0 .\end{cases}
$$




\section{APPENDIX D}

For general $g / L$, it is necessary to obtain a second term in Eq. (6.1) of order $k^{-1 / 2}$ relative to the first term [10]. We therefore rewrite Eq. (6.1) as

$$
\mathcal{P}_{11}^{L}(u)+\mathcal{Q}_{11}^{L}(u)=\frac{j k a}{2 L}+\frac{1}{a} \sum_{s=1}^{\infty}\left[\frac{e^{j p_{s}^{2} u / 2 k a^{2}}}{1-e^{-j p_{s}^{2} L / 2 k a^{2}}}+\frac{2 j k a^{2}}{L p_{s}^{2}}\right]+\frac{1}{a} \sum_{t=1}^{\infty} \frac{1}{p_{t}^{2}-1}\left[\frac{e^{j p_{t}^{2} u / 2 k a^{2}}}{1-e^{-j p_{t}^{2} L / 2 k a^{2}}}+\frac{2 j k a^{2}}{L p_{t}^{2}}\right] .
$$

Since the first bracket [] vanishes for large $k$ unless $p_{s}^{2}$ is of order $k a^{2} / L$, we can convert it to an integral. Specifically, we write

$$
w=\frac{1-j}{2} \frac{p_{s}}{a} \sqrt{\frac{L}{k}}, \quad \sum_{s=1}^{\infty} \Rightarrow(1+j) \frac{a}{\pi} \sqrt{\frac{k}{L}} \int_{0}^{\infty} d w .
$$

But the summand in the second bracket [] has an additional factor $\left(p_{t}^{2}-1\right)^{-1}$ which, for $p_{t}^{2} \sim k a^{2} / L$, reduces the contribution to Eq. (D1) by a factor of order $1 / k$, and can be neglected in Eq. (D1). Thus we have

$$
\mathcal{P}_{11}^{L}(u)+\mathcal{Q}_{11}^{L}(u)=-\frac{j k a}{2 L}-\frac{(1+j)}{\pi} \sqrt{\frac{k}{L}} \int_{0}^{\infty} d w\left[\frac{e^{-u w^{2} / L}}{e^{w^{2}}-1}-\frac{1}{w^{2}}\right] .
$$

Yokoya expresses the second term in Eq. (D1) as a power series in $u$ where the coefficients are $\zeta$ functions of half-integral argument and then proceeds to solve the integral equations numerically [10]. We shall instead assume that contributions to the integral in Eq. (D3) come primarily for small values of $w^{2}$, and expand in powers of $w^{2}$. Specifically, we write

$$
\begin{aligned}
\int_{0}^{\infty} d w\left[\frac{e^{-(u / L) w^{2}}}{e^{w^{2}}-1}-\frac{1}{w^{2}}\right] & \cong \int_{0}^{\infty} d w\left[\frac{\left(1-(u / L) w^{2}\right)}{w^{2}\left(1+w^{2} / 2\right)}-\frac{1}{w^{2}}\right] \\
& =-\left(\frac{u}{L}+\frac{1}{2}\right) \int_{0}^{\infty} \frac{d w}{1+w^{2} / 2}=-\frac{\pi}{\sqrt{2}}\left(\frac{u}{L}+\frac{1}{2}\right) .
\end{aligned}
$$

Thus Eq. (6.6) becomes

$$
-(1+j) \sqrt{\frac{k}{\pi}} \int_{0}^{z} \frac{d z^{\prime} e_{z}\left(z^{\prime}\right)}{\sqrt{z-z^{\prime}}}+\int_{0}^{g} d z^{\prime} e_{z}\left(z^{\prime}\right)\left[-\frac{j k a}{2 L}+\frac{(1+j)}{\sqrt{2}} \sqrt{\frac{k}{L}}\left(\frac{z-z^{\prime}}{L}+\frac{1}{2}\right)\right]=1,
$$

where the additional term in the kernel is of order $k^{-1 / 2}$ relative to the dominant term for large $k$.

We see from Eqs. (4.14) and (4.15) that the transverse impedance per period is given by

$$
\frac{Z_{x}(k)}{Z_{0}}=-\frac{1}{\pi k a^{3}} \int_{0}^{g} d z^{\prime} e_{z}\left(z^{\prime}\right) .
$$

The solution of Eq. (D5) can be written in the form

$$
e_{z}\left(z^{\prime}\right)=\frac{A}{\sqrt{z^{\prime}}}+\frac{B \sqrt{z^{\prime}}}{g} .
$$

Substituting Eq. (D7) into Eq. (D5) and performing the integrals leads to

$$
\begin{array}{r}
-(1+j) \sqrt{\pi k}\left[A+\frac{B z}{2 g}\right]-\frac{j k a \sqrt{g}}{L}\left(A+\frac{B}{3}\right)+\frac{1+j}{\sqrt{2}} \sqrt{\frac{k g}{L}}\left(\frac{2 z}{L}+1\right)\left(A+\frac{B}{3}\right)- \\
\frac{1+j}{\sqrt{2}} \sqrt{\frac{k g}{L}}\left(\frac{A}{3}+\frac{B}{5}\right) \frac{2 g}{L}=1 .
\end{array}
$$

Matching coefficients of the $z^{0}$ and $z^{1}$ terms leads to

$$
\begin{gathered}
A+\frac{(1+j)}{2} \sqrt{\frac{k g}{\pi}} \frac{a}{L}\left(A+\frac{B}{3}\right)-\sqrt{\frac{g}{2 L \pi}}\left(A+\frac{B}{3}\right)+\frac{g}{L} \sqrt{\frac{2 g}{\pi}}\left(\frac{A}{3}+\frac{B}{5}\right)=\frac{j-1}{2 \sqrt{\pi k}}, \\
\frac{B}{2 g}-\frac{1}{L} \sqrt{\frac{2 g}{L \pi}}\left(A+\frac{B}{3}\right)=0 .
\end{gathered}
$$


Setting

$$
A+B / 3=C,
$$

Eq. (D9) can be written as

$$
C\left[1-\frac{1}{\sqrt{2 \pi}}\left(\frac{g}{L}\right)^{1 / 2}+\frac{2}{3 \sqrt{2 \pi}}\left(\frac{g}{L}\right)^{3 / 2}+\frac{1+j}{2} \frac{a}{L} \sqrt{\frac{k g}{\pi}}\right]-\frac{B}{3}\left[1-\frac{8}{15 \sqrt{2 \pi}}\left(\frac{g}{L}\right)^{3 / 2}\right]=\frac{j-1}{2 \sqrt{\pi k}} .
$$

Using Eq. (D10), we then find

$$
\frac{1}{C}=-\left[\frac{1+j}{2} \frac{a}{L} \sqrt{\frac{k g}{L}}+\lambda\left(\frac{g}{L}\right)\right](1+j) \sqrt{\pi k}
$$

where

$$
\lambda(\xi)=1-\frac{\xi^{1 / 2}}{\sqrt{2 \pi}}-\frac{2 \xi^{3 / 2}}{3 \sqrt{2 \pi}}+\frac{16}{45 \pi} \xi^{3},
$$

where $\xi=g / L$. In Fig. 3 we present $g / L$ dependence of our approximate parameter $\lambda$ [given by Eq. (D14)] and the exact parameter obtained numerically following Yokoya's approach [10]. One can see that our analytically derived parameter $\lambda(g / L)$ is accurate to within a few percent. The admittance per period is therefore

$$
\begin{aligned}
Z_{0} Y_{x}(k) & =-\frac{\pi k a^{3}}{2 C \sqrt{g}} \\
& =\pi k^{2} a^{3}\left[\frac{j a}{2 L}+\frac{(1+j)}{2} \sqrt{\frac{\pi}{k g}} \lambda\left(\frac{g}{L}\right)\right] .
\end{aligned}
$$

We note that a more accurate expression for the parameter $\lambda(g / L)$ can be easily obtained if one performs a power series expansion of Eq. (D3), with the coefficients related to the Riemann $\zeta$ functions, and follows the steps similar to Eqs. (D7)-(D13). Note that keeping two terms in Eq. (D7) generates correctly only the first two coefficients for $\xi^{1 / 2}$ and $\xi^{3 / 2}$. Three terms are required to obtain the coefficient for $\xi^{5 / 2}$ correctly. Following these

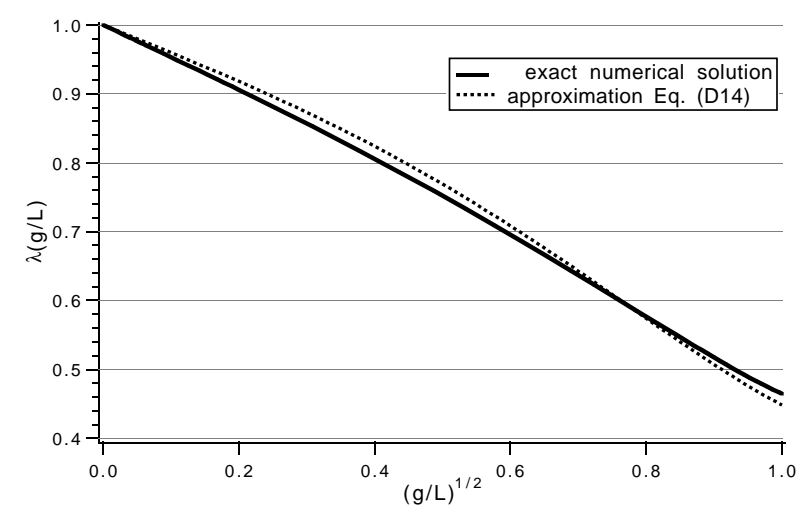

FIG. 3. Parameter $\lambda$ vs $g / L$. guidelines one obtains

$$
\begin{aligned}
\lambda(\xi) \cong & 1+\frac{\alpha_{0}}{\pi} \xi^{1 / 2}-\frac{\alpha_{1}}{3 \pi} \xi^{3 / 2}+\frac{13 \alpha_{2}}{45 \pi} \xi^{5 / 2} \\
& +\frac{8 \alpha_{1}^{2}}{45 \pi^{2}} \xi^{3}-O\left(\xi^{7 / 2}\right),
\end{aligned}
$$

where

$$
\begin{gathered}
\alpha_{0}=\zeta(1 / 2) \cong-1.4604, \quad \alpha_{1}=\frac{\zeta(3 / 2)}{2} \cong 1.3062, \\
\alpha_{2}=\frac{3 \zeta(5 / 2)}{8} \cong 0.5031,
\end{gathered}
$$

with

$$
\alpha_{m}=\frac{\zeta(m+1 / 2) \Gamma(m+1 / 2)}{m ! \Gamma(1 / 2)},
$$

where $\zeta$ denotes the Riemann zeta function. We also suggest the simple empirical form

$$
\tilde{\lambda}(\xi)=1+\left(\xi^{1 / 2} / \pi\right)\left(\alpha_{0}-\alpha_{1} \xi / 3+2 \alpha_{2} \xi^{2} / 5\right),
$$

which fits the exact numerical curve to within $1 \%$ for any $g / L$. In Fig. 4 we show the deviation of parameter $\lambda$ given by Eqs. (D19), (D16), and (D14) from the exact numerical solution [10].

Finally, because it is relatively straightforward to write an arbitrary number of terms in the series (D16), we checked Yokoya's conjecture [10] (inspired by Stupakov's calculation [11]) that

$$
\lambda(1)=\frac{-\zeta(1 / 2)}{\pi} .
$$

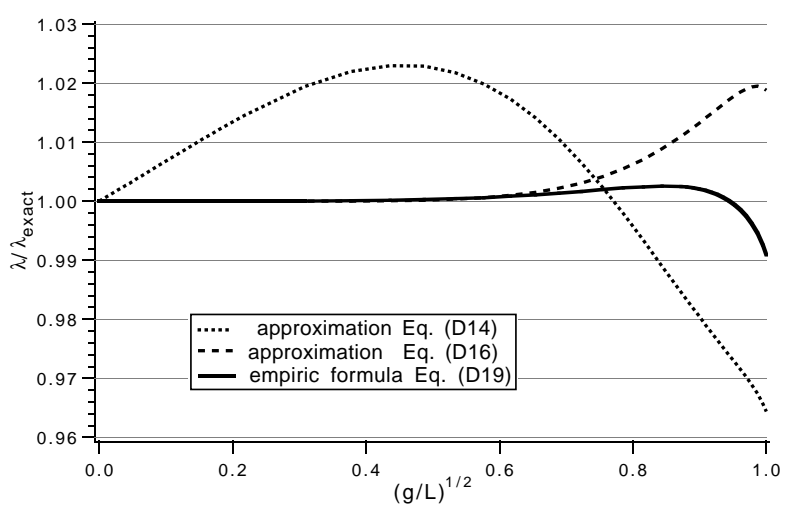

FIG. 4. Parameter $\lambda$ for different approximations. 
We found that, by retaining 80 terms in the series (D16), Eq. (D20) holds with a maximum relative error smaller than $5 \times 10^{-4}$.

[1] R. L. Gluckstern, Phys. Rev. D 39, 2773 (1989).

[2] R. L. Gluckstern, Phys. Rev. D 39, 2780 (1989).

[3] This calculation is similar to an earlier one for the high frequency smoothed longitudinal impedance [1].

[4] We shall avoid an ambiguity which later occurs by allowing $\lambda$ and $\mu$ to depart slightly from Eq. (3.4) to take into account the effect of a high but finite conductivity in the wall at $r=b$.

[5] At this point we note that, had we used $e_{\theta}\left(z^{\prime}\right)$ rather than $d e_{\theta}\left(z^{\prime}\right) / d z^{\prime}$ in Eq. (4.9), the corresponding kernel would have been $\left(z-z^{\prime}\right)^{-3 / 2}$, thus making that integral divergent. This has been avoided by using $d e_{\theta}\left(z^{\prime}\right) / d z^{\prime}$.

[6] We note at this point that the behavior of $e_{z}\left(z^{\prime}\right)$ near $z^{\prime}=0$ is expected to be $\left(k z^{\prime}\right)^{-1 / 3}$ for $k z^{\prime} \ll 1\left(z^{\prime}\right.$ small compared with the wavelength $2 \pi / k)$. Thus $\left(k z^{\prime}\right)^{1 / 2}$ in the denominator of Eq. (4.11) should be replaced by $\left(k z^{\prime}\right)^{1 / 2}+\operatorname{const}\left(k z^{\prime}\right)^{1 / 3}$, where const is $\mathcal{O}(1)$. Nevertheless, the dominant region for the integration over $z^{\prime}$ in Eq. (4.15) is for $k z^{\prime} \sim k g \gg 1$, and thus $\left(k z^{\prime}\right)^{1 / 2} \gg$ $\left(k z^{\prime}\right)^{1 / 3}$. It is for this reason that Eq. (4.11) does not accurately reflect the behavior for values of $k z^{\prime} \ll 1$, but this has no effect on the final result in Eq. (4.15).

[7] A. Chao, Physics of Collective Beam Instabilities in High Energy Accelerators (Wiley, New York, 1993).

[8] K. Bane and M. Sands, Part. Accel. 25, 73 (1990).
[9] $p_{s}$ and $p_{t}$ are given a small positive imaginary part to ensure the convergence of the sums in Eq. (5.12).

[10] K. Yokoya (private communication); also K.L.F. Bane and K. Yokoya, in Proceedings of the 1999 Particle Accelerator Conference (PAC99), New York (to be published).

[11] G. Stupakov, in Proceedings of the 1995 Particle Accelerator Conference, Dallas, Texas (IEEE, Piscataway, NJ, 1996), p. 3303.

[12] K. L.F. Bane, A. Mosnier, A. Novokhatskii, and K. Yokoya, in Proceedings of the European Particle Accelerator Conference, Stockholm, Sweden, 1998 (Institute of Physics, Bristol, UK, 1998).

[13] L. A. Vainshtein, Theory of Diffraction (Golden Press, Boulder, CO, 1969).

[14] B. Zotter and S. Kheifets, Impedances and Wakes in High-Energy Particle Accelerators (World Scientific, Singapore, 1998).

[15] R. L. Gluckstern, in Proceedings of the 1989 Particle Accelerator Conference, Chicago, IL (IEEE, Piscataway, NJ, 1989), p. 1157.

[16] S. S. Kurennoy and S. V. Purtov, Part. Accel. 34, 155 (1990).

[17] S. S. Kurennoy, Part. Accel. 39, 1 (1992).

[18] V. Balbekov, IHEP Report No. 93-55, 1993.

[19] S. S. Kurennoy (private communication).

[20] Note that we obtain $\mp j$, depending on whether $1-\epsilon$ lies inside or outside the unit circle. It is for this reason that we used the losses at $r=b$ to determine the appropriate sign in Eq. (C16). 